\title{
Sylviculture et activité collective longitudinale : un premier repérage
}

Silviculture and longitudinal collective activity: preliminary analysis

Marc-Éric Bobillier Chaumon, Pierre Ruyneau de Saint George, Bruno Cuvillier et Philippe Sarnin

\section{(2) OpenEdition}

1 Journals

Édition électronique

URL : http://journals.openedition.org/activites/265

DOI : $10.4000 /$ activites.265

ISSN : 1765-2723

Éditeur

ARPACT - Association Recherches et Pratiques sur les ACTivités

Référence électronique

Marc-Éric Bobillier Chaumon, Pierre Ruyneau de Saint George, Bruno Cuvillier et Philippe Sarnin, « Sylviculture et activité collective longitudinale : un premier repérage », Activités [En ligne], 11-1 | Avril 2014, mis en ligne le 15 avril 2014, consulté le 03 mai 2019. URL : http://journals.openedition.org/ activites/265 ; DOI : 10.4000/activites.265

\section{(c) (i) (9)}

Activités est mis à disposition selon les termes de la licence Creative Commons Attribution - Pas d'Utilisation Commerciale - Pas de Modification 4.0 International. 


\title{
Sylviculture et activité collective longitudinale : un premier repérage Marc-Éric Bobillier Chaumon
}

Université Lyon 2 (Lyon 2) - Institut de Psychologie - Laboratoire GRePS marc-eric.bobillier-chaumon@univ-lyon2.fr

\section{Pierre Ruyneau de Saint George}

CNAM Centre Régional de Lyon mail : saintgeorge.p@gmail.com

\section{Bruno Cuvillier}

Université Lyon 2 (Lyon 2) - Institut de Psychologie - Laboratoire GRePS bruno.cuvillier@univ-lyon2.fr

\section{Philippe Sarnin}

Université Lyon 2 (Lyon 2) - Institut de Psychologie - Laboratoire GRePS Philippe.Sarnin@univ-lyon2.fr

\begin{abstract}
Silviculture and longitudinal collective activity: preliminary analysis. We examine the case of forestry and show the reality of multigenerational forest management. This activity takes two forms: that of distributed work and that of cooperative longitudinal activity. In the case of distributed work, foresters apply working rules, which may or may not be explicit, collectively developed by and throughout History. In the case of cooperative activity, foresters use their knowledge of how the forest functions to optimize the future working situation and thus facilitate the work of their successors. In this case, cooperative work is based on a pragmatic and shared conception of a trajectory. This conception is developed through a form of controversy shaped by historical documents which have become intermediary objects.
\end{abstract}

KEY WORDS

forestry, work collective, collective work, trajectory

\section{1.- Contexte de l'étude et questions de recherche}

Amener des arbres forestiers à terme nécessite les soins conjugués de plusieurs générations de sylviculteurs. Ainsi, par exemple, le processus sylvicole qu'implique la production d'un sapin dure 100 à 150 ans. Ceci nous a amenés à nous interroger sur la question de la mise en œuvre d'une activité multi-générationnelle sylvicole. Nous nous sommes demandés, concrètement, si aïeux, grand-père, père et fils sylviculteurs ${ }^{1}$ travaillaient réellement de concert et, dans l'affirmative, par quels moyens.

Ce cas sort de l'ordinaire. La conception habituelle de l'organisation du travail s'appuie en

Pour ne pas alourdir le texte, nous avons opté pour le genre neutre, c'est-à-dire pour la forme masculine. Il est bien entendu qu'un sylviculteur, comme un aïeul ou un grand-père, un père ou un fils peut être d'un sexe ou d'un autre. 
effet sur une instance humaine dirigeante à qui revient le rôle de définir la tâche collective. Mais cette conception trouve ici une limite : qui serait en effet le dirigeant des sylviculteurs qui se succèdent pour la gestion d'une forêt donnée ? Qui en serait le « N+1» qui définirait la tâche sylvicole collective ? Reviendrait-il à l'un d'eux de définir une finalité forestière, par exemple «faire du bois d'œuvre » ou «entretenir un espace de biodiversité » à laquelle devraient se conformer les successeurs? Mais d'où tiendrait-il une telle légitimité ? Comment pourrait-il procéder pour que son cap soit maintenu après, et longtemps après, sa disparition? D'autre part, faute de «N+1», il ne peut y avoir de prescrit hiérarchique préétabli coordonnant les actions des uns par rapport à celles des autres et, faute de contemporanéité, cette coordination ne peut pas davantage s'ajuster par la parole ou le geste. Comment, alors, les actions sylvicoles peuvent-elles être orchestrées ?

Le cas du projet multi-générationnel, projet dont la durée nécessite la conjugaison de plusieurs générations d'intervenants, nous semble tout à fait inédit pour la psychologie du travail et l'ergonomie. Pourtant, de tels projets existent. Schmidt (cité par Barthe, \& Quéinnec, 1999), par exemple, mentionne la construction architecturale et la recherche scientifique.

Notre proposons ici un premier repérage concernant le cas de la sylviculture et nous nous fixons deux objectifs scientifiques. Le premier est une caractérisation de situations multigénérationnelles du travail sylvicole. Il s'agit, concrètement, de s'interroger sur la réalité d'une activité collective sylvicole impliquant une lignée (aïeux, grand-père, père et fils) de sylviculteurs. Le second objectif est de mettre à jour des éléments qui participent à l'organisation d'une activité collective séculaire particulière : celle qui se caractérise par une absence de prescrit hiérarchique et d'ajustement mutuel par le geste ou la parole.

\section{1.- Les caractéristiques de l'activité de sylviculture}

L'activité de sylviculture consiste à planifier et prescrire des interventions forestières telles que les reboisements (choix des essences, de la densité de plantation...), les éclaircies ${ }^{2}$ (désignation des arbres à prélever...), les coupes, la mise en place d'infrastructures (pistes, accès, zones de stockage....). La sylviculture correspond ainsi à "l'activité décisionnelle » faisant appel à «des dimensions immatérielles liées aux processus cognitifs en jeu (intelligence de la situation, conception de solutions et choix d'une solution satisfaisante, évaluation)» qu'ont identifiée Cerf et Maynard (2006, p.24). Il s'agit de la phase préparatoire qui précède toute «action de transformation des processus agroécologiques ». Elle se réalise dans un couplage entre plan d'aménagement (conception, formalisée ou non, d'un processus de travail rythmé par les interventions sylvicoles) et interventions sylvicoles (éclaircies, plantations, reboisement par régénération naturelle...). Par ailleurs, pour les forêts qui dépassent une certaine superficie, le plan d'aménagement (appelé «Plan Simple de Gestion ») résulte de compromis sociaux, car il doit être agréé par le CRPF (Centre Régional de la Propriété Forestière) qui prône une sylviculture "politiquement correcte » parfois à l'encontre des politiques sylvicoles souhaitées par le sylviculteur. Dans le cas de groupements forestiers ou de forêts en indivision, le sylviculteur doit aussi se coordonner avec ses associés parmi lesquels se trouvent des «écolos» et des «financiers» pour reprendre les termes des sylviculteurs. Il résulte de tout cela que les actions des sylviculteurs, comme l'ont observé Pueyo et Zara-Meylan (2012) lorsqu'il s'agit de «sortir les plantes », "sont moins situées dans l'immédiateté que dans le processus de travail et la dynamique sociale de son activité » (p. 16).

Les conditions de l'activité sylvicole semblent également proches de celles de la conduite d'une exploitation agricole, conduite marquée par l'importance des régulations (Cellier, \& Marquié, 1980). Plusieurs raisons à cela. D'abord le sylviculteur doit composer avec des conditions environnementales qu'il ne peut ni maîtriser ni anticiper. Des évènements naturels

2 Les termes techniques sont répertoriés dans un glossaire en fin d'article. 
et climatiques (tempêtes, sécheresses, neiges excessives, maladies, pression du gibier...), des décisions administratives (durcissements de réglementations...), des évolutions économiques du marché du bois, des évènements conjoncturels (pénurie de plants, pannes de matériel, désistements d'Entrepreneurs de Travaux Forestiers, terrain qui ne permet pas un débardage aisé...) l'obligent à mettre en place opportunément des interventions sylvicoles non planifiées, à les décaler ou à les modifier.

Ainsi, l'activité sylvicole, bien qu'elle soit consignée comme une procédure d'intervention (par un plan d'aménagement), apparaît comme une tâche qui ne peut être totalement prescrite. Elle s'inscrit dans un environnement instable et complexe (social, administratif, économique, écologique...) qui va affecter les conditions de sa réalisation.

Dans la mesure où nous faisons l'hypothèse d'une situation multi-générationnelle de travail, il est nécessaire de caractériser ces dimensions collectives et de voir comment celles-ci peuvent s'articuler dans le temps, optique qui nous amène vers le concept de l'articulation de trajectoire.

\section{2.- L'activité collective}

Nous prenons l'activité collective comme «une combinaison entre le travail collectif et le collectif de travail» (Caroly, 2010, p. 113). Le travail collectif couvre les fonctions de régulation de la production et de l'efficience. En d'autres termes, le travail collectif correspond à l'activité effective mise en œuvre pour réaliser une tâche. Mais une situation collective de travail ne se limite pas à un travail collectif. Elle se colore aussi des effets liés aux modes de solidarité des membres impliqués dans une œuvre commune.

Dans le cas général, la structuration du travail collectif est pensée par l'organisation. Des concepteurs définissent les tâches qu'ils jugent nécessaires pour la réalisation d'un objectif. Il s'agit du travail prescrit. Mais l'individu en situation de travail s'éloigne de ce qu'on lui demande de faire ou de ce qu'on attend de lui, car il prend en compte les exigences de son activité, les opportunités et les circonstances de son travail (Valléry, \& Amalberti, 2006). Les modes opératoires réellement mis en oeuvre s'éloignent ainsi des procédures prédéfinies et la tâche elle-même est susceptible d'être redéfinie par les exécutants (Leplat, 2011). C'est ainsi que le travail réel se différencie du travail prescrit. Il en est de même sur le plan collectif. Le travail collectif (réel) autour d'une œuvre commune s'éloigne du travail collectif prescrit. Ce décalage suppose une activité collective d'organisation de la part des acteurs eux-mêmes. La coopération en est une forme particulière dans laquelle «le traitement des interférences par un ou plusieurs opérateurs est effectué de manière à faciliter les activités des coéquipiers » (Barthe, \& Quéinnec, 1999, p. 674).

Cette réorganisation du travail collectif par les acteurs eux-mêmes peut se réaliser sous la forme de l'ajustement mutuel interpersonnel. Les acteurs se concertent alors, négocient ou délibèrent sur ce qu'il convient de faire. Ils définissent des règles de travail. C'est la dimension «équipe de travail». Les exécutants, capables de positionner leurs tâches par rapport à celles des autres, sont alors en situation de collaboration.

L'activité collective d'organisation du travail collectif peut se traduire par une matrice plus élaborée qui pré-organise l'action individuelle à travers des règles de métier ou des spécifications qui fondent le genre professionnel.

Les règles de métier sont des règles qui définissent des façons de travailler, de se comporter vis-à-vis d'autrui ainsi que les significations langagières et les valeurs éthiques au sein d'un corps de métier (Molinier, 2006). Elles sont élaborées par la communauté de métier. Le genre professionnel (Clot, 1999) résulte d'un ensemble de spécifications complémentaires, partagées et sous-entendues "entrées dans la chair des professionnels », qui "préorganisent leurs opérations et leur conduite » (p. 34, Clot, op. cit). Le genre professionnel se révèle à travers des attendus implicites. 
Règles de métier et spécifications génériques apparaissent comme des vecteurs et des déterminants de l'activité. Ils sont «extérieurs » à l'individu et apparaissent ainsi comme des «faits sociaux», c'est-à-dire comme des «manières d'agir, de penser et de sentir, extérieures à l'individu, et qui sont douées d'un pouvoir de coercition en vertu duquel [ces faits sociaux] s'imposent à lui »(Durkheim, 1895, p. 102). L'existence de tels «faits sociaux » permet de distinguer le collectif de travail par rapport à l'équipe de travail.

En sylviculture, le travail collectif apparaît donc lignager. L'entretien d'une forêt résulte en effet d'un ensemble de tâches éclatées dans le temps (passé, présent et futur) et dans des mains de sylviculteurs qui se succèdent. Ceux-ci, pour rendre leurs interventions pertinentes au regard du développement de la forêt, doivent pouvoir, à un moment donné, relier et coordonner les tâches. Cet aspect nous renvoie au concept d'articulation de trajectoire.

\section{3.- La coordination des interventions : l'articulation de trajectoire}

Le concept d'articulation souligne comment les personnes qui ne partagent a priori ni les mêmes objectifs, ni les mêmes définitions de la situation déploient néanmoins «un travail supplémentaire " "pour que les efforts de l'équipe soient finalement plus que l'assemblage chaotique de fragments épars de travail »(Strauss, 1992, p. 191). Or cette problématique est bien présente en sylviculture puisque chaque génération de sylviculteurs peut avoir ses propres objectifs et des finalités divergentes, par exemple sur des enjeux économiques ou écologiques.

Pour Strauss (1992), le travail collectif s'inscrit dans une négociation des rôles de chacun, des règles, des objectifs. Cette négociation dessine une trajectoire autour de laquelle s'articulent les différents acteurs en fonction de leurs rôles, prérogatives, perspectives. Le travail d'articulation est alors ce travail de reliaison qui se fait entre les différents interlocuteurs engagés dans un objectif commun, notamment lorsque le travail est réparti entre différents acteurs, temporalités et dans différents espaces. Au sens initial de Strauss (op. cit), et rapporté au domaine sylvicole, la trajectoire fait non seulement référence à l'évolution naturelle du développement agronomique de la forêt (le process), mais s'attache aussi à l'organisation du travail qu'il faut déployer pour en suivre le cours (le processus) et en tirer ainsi une certaine cohérence (par le travail d'articulation).

L'articulation est ainsi « un travail sur le travail » nécessaire pour coordonner des activités de plusieurs groupes professionnels et de plusieurs ordres de tâches, afin que la trajectoire effective aboutisse à un résultat satisfaisant. "Ce travail composite de mise en cohérence, d'articulation des actions de chacun des professionnels qui génère tant de difficultés et dont la qualité va croissante n'est ni formalisé, ni explicité. [...] Non reconnu, il est l'impensé du travail, le "parasite" du "vrai" travail à savoir celui qui figure dans la définition de la fonction » (Grosjean, \& Lacoste, 1999, p. 172). L'articulation permet ainsi l'assemblage, le maintien ensemble d'éléments séparés qui tout en restant distincts peuvent se mouvoir aisément les uns par rapport aux autres (Schmidt, 2002). Elle permet aussi la bonne compréhension et donc la qualité de la communication par l'intelligibilité qu'elle favorise.

Dans ces conditions, on peut supposer qu'il existe un travail d'articulation pour donner un sens et une finalité globale à des activités de sylviculture éclatées dans le temps (entre des activités d'exploitation passées, présentes, mais aussi futures) afin qu'elles ne restent pas isolées, sans lien les unes avec les autres.

\section{4.- Questions de recherche}

L'objectif de notre recherche consiste donc à s'interroger sur l'existence et les modalités d'un travail collectif multi-générationnel dans l'activité de sylviculture. Il s'agit d'identifier les formes de solidarité entre sylviculteurs successifs et de déterminer sur quoi se base une lignée de sylviculteurs pour arriver à travailler de concert. Cette question nous amènera à porter une attention particulière à la (ré)-élaboration multi-générationnelle des règles (car 
elle signe une activité collective d'organisation) ainsi qu'au travail d'articulation qui s'y déploie.

Plus précisément, nous supposons que la situation multi-générationnelle de travail sylvicole peut prendre deux formes archétypale d'organisation collective :

- Celle d'un travail distribué. Dans ce cas, les sylviculteurs appliquent des règles de métier et des spécifications génériques collectivement élaborées par la communauté de métier dans, et par, l'Histoire. De ce mode d'élaboration, il résulte un agencement pertinent de ces règles au regard du développement d'une forêt. Il en résulte une gouvernance appropriée de l'action du sylviculteur.

- Celle d'une activité coopérative et articulée. Dans ce cas les sylviculteurs exploitent, en connaissance de cause, des savoirs sur le fonctionnement de leur forêt pour optimiser la situation de travail future et ainsi faciliter ou rendre plus efficientes les activités de leurs successeurs.

\section{2.- Méthodologie de recherche}

L'accès à une activité collective multi-générationnelle pose, dans son principe, une difficulté méthodologique. Comment en effet rendre compte d'un processus séculaire, fragmenté dans le temps? Pour approcher cette complexité, nous avons opté pour une démarche par triangulation de données en travaillant d'abord à partir (i) de témoignages de sylviculteurs (entretiens semi-directifs) que nous avons confrontés (ii) aux traces de l'activité sylvicole contemporaines et passées - consignées sur des documents d'archives, sur des matériels et des objets de l'activité ainsi qu'aux (iii) pratiques réelles de sylviculture (par des observations en situation d'intervention forestière).

\section{1.- L'échantillon étudié}

Nous nous sommes orientés vers des sylviculteurs réellement engagés dans la sylviculture, c'est-à-dire devant réaliser des opérations forestières dictées par des contraintes de terrain, des raisons économiques ou encore par des traditions historiques et familiales. La réalisation d'éclaircies étant propre à la sylviculture (Cardon, 1999), nous avons retenu 4 propriétaires forestiers qui réalisaient ce type de tâche. Ils représentent un spectre assez large du point de vue des âges, des activités, des superficies de forêt... (cf. tableau 1). Ces 4 sylviculteurs ont également été sollicités parce qu'ils avaient tous un rapport particulier à la forêt : l'un (P) était considéré comme un sylviculteur actif par ses pairs, un autre (C) s'est mis tardivement à la sylviculture sans bénéficier d'une imprégnation forestière préalable ; un troisième (L) avait anticipé la transmission de la gestion forestière ; le dernier (G), issu d'une famille de sylviculteurs, représentait le cas archétypal de l'activité lignagère coopérative. Le tableau suivant synthétise les caractéristiques de cet échantillon.

En dépit des différences d'âges, de situations professionnelles et historiques, de tailles de forêts, de modes d'entrée dans la sylviculture, de finalités investies dans la forêt, tous montrent une implication dans l'activité sylvicole. On note cependant que les sylviculteurs interrogés cumulent des caractéristiques qui les éloignent des cas habituellement traités en ergonomie ou en psychologie du travail. Les objets de leur travail sont des biens hérités et la sylviculture n'est ni leur activité principale ni leur ressource économique principale. Les gains octroyés ne permettent pas d'en vivre, mais fournissent néanmoins un revenu d'appoint pour deux d'entre eux $(G \& C)$. Cela peut d'ailleurs aussi constituer une des motivations de leur engagement. Ces spécificités ne sont pas neutres dans leurs manières d'appréhender la sylviculture et il ne faut pas être étonné de voir le cas des sylviculteurs interrogés s'éloigner de certains des résultats de Schepens (2007). Dans son étude sur les ETF (Entrepreneur de Travaux Forestiers), parmi lesquels il range les sylviculteurs à titre professionnel, Schepens (2007) affirme ainsi : « "Si pendant longtemps nous avons cherché la famille derrière chaque ETF persuadé qu'il s'agissait d'activités transmises de père en 
fils, nous pouvons maintenant l'assurer : il ne se construit pas de dynasties de forestiers... on ne devient ETF de père en fils que dans moins de $10 \%$ des cas» (p. 103). On voit d'ailleurs le frère de L, travailleur forestier, préférer prendre la ferme familiale et laisser les bois familiaux à son frère.

\begin{tabular}{|c|c|c|c|c|}
\hline & Sylviculteur L & $\mathbf{P}$ & G & $\mathbf{C}$ \\
\hline Âge & 73 ans & 80 ans & 45 ans & 49 ans \\
\hline Origine & $\begin{array}{l}\text { Issu d'une famille } \\
\text { d'agriculteurs }\end{array}$ & $\begin{array}{l}\text { Fils de travailleur } \\
\text { forestier, petit-fils } \\
\text { d'agriculteurs }\end{array}$ & $\begin{array}{c}\text { Issu de familles } \\
\text { notables de la } \\
\text { campagne }\end{array}$ & $\begin{array}{c}\text { Fils d'un employé } \\
\text { de bureau, petit-fils } \\
\text { d'agriculteurs }\end{array}$ \\
\hline $\begin{array}{c}\text { Situation } \\
\text { professionnelle }\end{array}$ & $\begin{array}{l}\text { Employé de } \\
\text { bureau à la } \\
\text { retraite }\end{array}$ & $\begin{array}{c}\text { Employé de } \\
\text { bureau à la retraite }\end{array}$ & $\begin{array}{l}\text { Ingénieur, agrégé } \\
\text { de mathématiques }\end{array}$ & $\begin{array}{l}\text { Directeur de bureau } \\
\text { de poste }\end{array}$ \\
\hline $\begin{array}{l}\text { Implication dans des } \\
\text { institutions sylvicoles }\end{array}$ & $\begin{array}{l}\text { Impliqué lors de } \\
\text { la mise en place } \\
\text { de la coopérative } \\
\text { forestière }\end{array}$ & $\begin{array}{c}\text { Autrefois } \\
\text { administrateur } \\
\text { pour un syndicat } \\
\text { des propriétaires } \\
\text { forestiers }\end{array}$ & Non. & $\begin{array}{l}\text { Actuellement un } \\
\text { des relais du } \\
\text { syndicat des } \\
\text { propriétaires } \\
\text { forestiers }\end{array}$ \\
\hline Superficie de la forêt & Quelques ha & $20 \mathrm{ha}$ & 400 ha & $35 \mathrm{ha}$ \\
\hline Origine de la forêt & $\begin{array}{l}\text { Plantation de } \\
\text { résineux achetée } \\
\text { par le père. }\end{array}$ & $\begin{array}{c}\text { « Elle vient de } \\
\text { très loin » du côté } \\
\text { maternel }\end{array}$ & $\begin{array}{c}\text { Lointaine (400 } \\
\text { ans). } \\
3000 \text { ha partagés au } \\
\text { fil des successions }\end{array}$ & $\begin{array}{l}5 \text { ha constitués par } \\
\text { son grand-père } \\
\text { maternel sur des } \\
\text { terrains agricoles }\end{array}$ \\
\hline Mode de propriété & Pleine propriété & Pleine propriété & $\begin{array}{c}\text { Groupement } \\
\text { forestier familial }\end{array}$ & $\begin{array}{c}\text { Groupement } \\
\text { forestier familial }\end{array}$ \\
\hline Succession & $\begin{array}{c}3 \text { filles peu } \\
\text { intéressées } \\
1 \text { petit-fils sur } \\
\text { lequel il fonde des } \\
\text { espoirs }\end{array}$ & $\begin{array}{c}2 \text { filles peu } \\
\text { intéressées } \\
2 \text { petites-filles } \\
\text { «pétries » en } \\
\text { forêt }\end{array}$ & $\begin{array}{c}\text { Pas d'enfant } \\
\text { Pas de neveux } \\
\text { montrant } \\
\text { actuellement un } \\
\text { intérêt }\end{array}$ & $\begin{array}{l}\text { Pas d'enfant } \\
\text { montrant } \\
\text { actuellement un } \\
\text { intérêt }\end{array}$ \\
\hline $\begin{array}{c}\text { Proximité } \\
\text { géographique }\end{array}$ & $\begin{array}{l}\text { Habite à } \\
\text { proximité de la } \\
\text { forêt }\end{array}$ & $\begin{array}{c}\text { Habite à } \\
\text { proximité de la } \\
\text { forêt }\end{array}$ & $\begin{array}{l}\text { Habite à } 800 \mathrm{~km} \text { de } \\
\text { la forêt }\end{array}$ & $\begin{array}{l}\text { Habite à proximité } \\
\text { de la forêt }\end{array}$ \\
\hline $\begin{array}{l}\text { Eléments significatifs } \\
\text { d'Histoire en lien avec } \\
\text { la forêt. }\end{array}$ & $\begin{array}{l}\text { Vers } 15 \text { ans, sous } \\
\text { l'aile de son frère } \\
\text { aîné, a dû abattre } \\
\text { ses premiers } \\
\text { arbres pour faire } \\
\text { face à de graves } \\
\text { problèmes } \\
\text { économiques. }\end{array}$ & $\begin{array}{l}\text { Père landais qui } \\
\text { émigra dans le } \\
\text { Lyonnais, avec } \\
\text { son oncle, ses } \\
\text { outils, "et toute } \\
\text { son expérience de } \\
\text { la forêt landaise» }\end{array}$ & $\begin{array}{l}\text { Déboires résultant } \\
\text { d'une époque } \\
\text { pendant laquelle la } \\
\text { gestion forestière a } \\
\text { été déléguée }\end{array}$ & $\begin{array}{c}\text { Choix de son } \\
\text { grand-père de } \\
\text { transformer des } \\
\text { terrains agricoles en } \\
\text { bois }\end{array}$ \\
\hline $\begin{array}{l}\text { Finalités investies dans } \\
\text { la forêt. (Inspirées de } \\
\text { la typologie de Brun, } \\
\text { \& Mallein, 2009) }\end{array}$ & $\begin{array}{c}\text { Placement, } \\
\text { épargne et } \\
\text { visée d'une } \\
\text { notabilité locale. }\end{array}$ & $\begin{array}{l}\text { Constitution d'un } \\
\text { espace de liberté } \\
\text { et de bien-être non } \\
\text { coûteux }\end{array}$ & $\begin{array}{l}\text { Production de } \\
\text { revenus dans le } \\
\text { respect de } \\
\text { l'environnement. } \\
\text { Optique gestion } \\
\text { durable. }\end{array}$ & $\begin{array}{c}\text { Objectifs } \\
\text { économiques dans } \\
\text { le respect de } \\
\text { l'environnement. } \\
\text { Optique gestion } \\
\text { durable. }\end{array}$ \\
\hline $\begin{array}{l}\text { Mode d'entrée dans } \\
\text { l'activité de } \\
\text { sylviculture }\end{array}$ & $\begin{array}{c}\text { Imprégnation à } \\
14 / 15 \text { ans } \\
\text { par son frère }\end{array}$ & $\begin{array}{c}\text { Imprégnation à } \\
14 / 15 \text { ans par son } \\
\text { père }\end{array}$ & $\begin{array}{l}\text { Imprégnation à } \\
14 / 15 \text { ans par son } \\
\text { père }\end{array}$ & $\begin{array}{c}\text { Découverte à } 22 \\
\text { ans }\end{array}$ \\
\hline $\begin{array}{c}\text { Exécution de travaux } \\
\text { forestiers } \\
\text { (bucheronnage, } \\
\text { débardage...) }\end{array}$ & $\begin{array}{l}\text { Oui, autrefois tous } \\
\text { les travaux } \\
\text { forestiers. }\end{array}$ & $\begin{array}{c}\text { Travaux } \\
\text { d'entretien } \\
\text { (élagage...) }\end{array}$ & Non & Oui, parfois. \\
\hline $\begin{array}{l}\text { Enjeux forestiers } \\
\text { prédominants }\end{array}$ & $\begin{array}{l}\text { Enjeux affectifs et } \\
\text { familiaux }\end{array}$ & $\begin{array}{l}\text { Enjeux affectifs et } \\
\text { familiaux }\end{array}$ & $\begin{array}{c}\text { Enjeux } \\
\text { économiques } \\
\text { (revenus réguliers) }\end{array}$ & $\begin{array}{c}\text { Enjeux } \\
\text { économiques } \\
\text { (défiscalisation) }\end{array}$ \\
\hline $\begin{array}{l}\text { Dernière opération } \\
\text { effectuée en forêt }\end{array}$ & Éclaircie & Dépressage & Éclaircie & Dépressage \\
\hline
\end{tabular}

Tableau 1 : Caractéristiques des sylviculteurs interrogés

Table 1: Characteristics of the foresters interviewed

Enfin, s'il s'agit là d'éléments «contextuels» au regard de notre objet d'étude (caractérisation de situations multi-générationnelles de travail, identification de modes 
structurants), nous avons bien conscience que ces différents facteurs socio-professionnels devraient être davantage pris en compte pour mieux cerner les diversités de pratique et d'engagement dans l'activité sylvicole. Cependant les données dont nous disposons ne nous permettent pas d'aller plus loin dans ces analyses individuelles. Cela fera d'ailleurs l'objet d'une des pistes de recherche proposée en conclusion.

\section{2.- Méthodes déployées}

\subsection{1.- Les entretiens réalisés}

Nous avons procédé à des entretiens semi-directifs et avons pris les règles de travail comme point d'entrée. Nous nous sommes tournés vers la question de leurs (ré)-élaborations, car celles-ci signent une activité organisationnelle collective. Nous avons conçu nos guides d'entretien autour de trois thèmes principaux : (1) L'histoire de la personne et de son parcours «sylvicole» (arrivée dans le métier, tradition/héritage familial, représentation de l'activité). (2) L'observation du rapport à la règle. Nous avons cherché à identifier des règles autour desquelles les sylviculteurs interrogés organisaient leurs actions ainsi qu'à sonder l'existence de (ré)-élaborations. (3) Les conditions effectives de réalisation de l'activité. Nous avons taché d'orienter le récit sur le réel non exceptionnel, c'est-à-dire sur des pratiques routinières et intégrées. Dans cette optique, nous avons demandé aux interviewés de parler de la dernière opération sylvicole réalisée et de décrire la prochaine opération prévue.

Les entretiens d'1h30 en moyenne et se sont déroulés à domicile. Ils ont été enregistrés par magnétophone et ont fait l'objet d'une transcription intégrale pour permettre une analyse approfondie et comparative.

\subsection{2.- Analyse des traces de l'activité}

Il s'est agi de recueillir toutes les traces matérielles visibles de l'activité et de sa transmission, en tant qu'elles peuvent être un support au travail et/ou témoigner du déroulement de celui-ci. En particulier :

- Des traces écrites : notes, annotations manuscrites sur documents et manuels, choix des revues ou manuels utilisés, agendas, comptes rendus des réunions (assemblées générales), plans d'aménagement forestier («Plans Simples de Gestion »).

— Des traces matérielles de l'activité. Nous nous sommes rendus sur les lieux des dernières opérations sylvicoles de trois des sylviculteurs interrogés pour une présentation commentée. Nous avons ainsi relevé les traces d'activité qui apparaissaient sous la forme reconnaissable de plantations, d'arbres élagués, de souches, de marquages, de protections contre le gibier, de pistes... Nous demandions aux personnes d'expliquer l'intérêt et l'origine de ces éléments dans son activité.

— Les archives: une facette de l'activité du sylviculteur est d'établir un plan d'aménagement. Aussi, nous avons consulté divers documents (agenda, archives) dans lesquels étaient consignés des diagnostics, des travaux forestiers prévus et commandés, des retours d'expérience, des stratégies d'exploitation... Autant d'éléments qui nous permettent d'accéder à une part du travail réel.

\subsection{3.- Observations d'interventions et des pratiques sylvicoles}

Nous avons observé 4 situations complémentaires d'interventions sylvicoles en train de se réaliser. Celles-ci réunissaient $\mathrm{S}$ et son bûcheron-garde-forestier à temps partiel. Il s'agissait de quatre situations typiques de l'activité de gestion d'un domaine forestier.

L'objet de la première situation de travail (octobre 2012) était de préparer le marquage pour une éclaircie sur 2 à 3 ha d'une plantation de sapins. L'opération a duré 2 heures en forêt et s'est terminée par un débriefing entre le bûcheron et $\mathrm{S}$ au domicile de ce dernier. La deuxième situation (novembre 2012) était la phase de marquage des arbres à prélever. Elle a duré 3 heures. La situation suivante (novembre 2011) était une opération de contrôle sur un 
dépressage en cours sur une plantation de 2 ha en douglas de 10 ans. Cette opération a duré $1 \mathrm{~h} 30$ en forêt. La dernière situation (octobre 2012) était la réception de travaux. Il s'agissait pour le sylviculteur de valider la réalisation d'une éclaircie ainsi que l'aménagement d'une piste de débardage et d'une zone de stockage.

L'objectif de ces observations (par papier-crayon) était d'identifier les pratiques mises en oeuvre dans le cadre des interventions sylvicoles, les ressources mobilisées pour mener à bien ces opérations, les interactions entre les différents acteurs présents et les difficultés rencontrées. À l'issue de chaque intervention, le sylviculteur était invité à commenter des évènements observés par verbalisation consécutive (Hoc, \& Leplat, 1983). Il était alors amené à justifier ses actions et ses décisions, à faire part de ses interrogations et de son « vécu » de la situation.

\section{3.- Méthode d'analyse des résultats}

Nous avons commencé par exploiter les entretiens semi-directifs par une analyse thématique transversale des corpus sur la base des thèmes suivants : faits sociaux à périmètre lignager, sentiment d'appartenance lignagère, gestion des finalités investies dans la forêt et (ré)élaboration de règles. Nous avons ensuite vérifié la cohérence entre le déclaratif et le réel par l'analyse des données matérielles et des observations recueillies.

\section{3.- Présentation et analyse des résultats : réalité et formes d'une situation multi-générationnelle de travail}

\section{1.- Un travail collectif dans l'activité sylvicole?}

Notre cadre théorique nous a conduits à définir le travail collectif comme des interventions coordonnées et articulées d'opérateurs différents en vue d'une œuvre commune. La question de ce travail collectif soulève dès lors 2 points : celui de l'interdépendance d'activités et celui de l'œuvre commune.

a) L'interdépendance d'activités se manifeste sous 2 aspects : d'abord, la production d'un bel arbre suppose des soins qui s'étalent sur plusieurs générations de sylviculteurs. Les travaux des uns et ceux des autres doivent donc se compléter, malgré leur temporalité différente. En l'occurrence, lors de notre visite sur leurs parcelles, $\mathrm{P}$ et $\mathrm{G}$ nous ont présenté certaines de leurs opérations sylvicoles en les positionnant comme étant la suite des réalisations de leurs prédécesseurs. Cette configuration se rattache à celle du travail collectif par rotations. Par ailleurs, toute action de sylviculture modifie la forêt, c'est-àdire les conditions d'exercice de l'activité future, d'où une interférence sur les activités à venir.

b) En ce qui concerne le partage d'un objectif commun, il ne faut pas, comme le rappellent, Grosjean et Lacoste (1999), se limiter à une acception trop forte de l'exigence d'un but commun. Il n'est pas rare, en effet, de voir se révéler des conflits de buts lors de la confrontation au réel. Nous considérons que cette situation est alors marquée par un déficit de «sentiment partagé de similitude » (Zeleny, 1939 ; cité par Anzieu, \& Martin, 1968 , p. 115) et partant, par une atteinte au «moral». Le terme «moral» est pris ici dans le sens de la dynamique des groupes (Anzieu, \& Martin, 1968), c'est-à-dire dans le sens d'un affect du registre de la cohésion.

Or, en sylviculture, l'existence d'une « œuvre commune » n'est pas assurée, car à un sylviculteur naturaliste, davantage porté sur des valeurs écologiques, peut succéder un sylviculteur de type «industriel», ou inversement. L'actualisation d'un tel risque pourrait produire des cadres d'activité incompatibles entre eux, qui, s'ils se succédaient, entraîneraient l'échec des uns et des autres.

Des effets de «moral» provoqués par une cohésion incertaine autour de finalités sylvicoles 
apparaissent chez les 4 sylviculteurs interrogés. Pourrait-il d'ailleurs en être autrement puisque, pour un sylviculteur, tout travail perd son sens s'il n'est pas poursuivi ? L, par exemple, exprime une plainte du registre de la cohésion autour de finalités investies dans la forêt lorsqu'il regrette que ses «enfants n'apprendront sans doute jamais à lire la forêt ». Ses successeurs sont alors vus comme des successeurs sans enjeux forestiers.

Nos résultats confirment l'existence de différences substantielles dans les finalités investies dans la forêt. P apparaît ainsi plutôt comme un artiste. Lors des observations de terrain, il montre les feuillus qu'il privilégie pour «satisfaire un peu tout le monde, satisfaire le paysage ». Il rejette la monoculture, s'apprête à planter du pin landais en souvenir de son père. Il cherche avant tout à construire une forêt dans laquelle on se sente bien, qui ait du sens, qui réponde à des valeurs familiales et écologiques. G, en revanche, est un industriel : la forêt doit être organisée de telle sorte qu'elle génère "des recettes suffisantes pour pouvoir faire vivre l'activité et distribuer un dividende aux associés» et ce, de façon régulière dans le temps.

Cependant, on constate bien une communauté de visée au sein des communautés lignagères. Par exemple, dans les archives de G, les premiers écrits concernant sa forêt datent de 1905. Ils rapportent l'histoire de la propriété depuis 1780, avec les noms des propriétaires, les partages effectués et le type de gestion sylvicole pour la période de 1869 à 1899. Cela représente près de 140 ans de sylviculture, soit une génération de résineux. Ce sont 9 gestionnaires de la forêt qui se sont ainsi succédés. Tous se présentent avec une optique du type «gestion durable»: "Suivant la formule consacrée, l'aménagement a pour but d'assurer la perpétuité de la forêt et son rendement soutenu » écrit un gérant (Notes de 1958, archives de la forêt de G).

Mais il ne pourrait s'agir là que d'une inertie, car la «visée » d'une lignée peut se transformer. Ainsi, $\mathrm{L}, \mathrm{P}$ et $\mathrm{C}$ sont les premiers de leurs lignées à procéder à des éclaircies, tâches qui distinguent la gestion sylvicole par rapport à la gestion paysanne de la forêt (Cardon, 1999). On observe alors une transformation des finalités investies dans la forêt, celle-ci perdant son statut de ressources domestiques et de «caisse d'épargne » pour prendre celui d'une production à commercialiser. Il apparaît donc que si une finalité a été constante dans une lignée pendant de nombreuses générations (la finalité «forêt paysanne »), elle peut cependant être l'objet d'un changement.

Il est cependant difficile de parler ici de tâche collective, car rien d'ex ante n'aimante le travail collectif.

\section{2.- Un collectif lignager?}

Comme nous l'avons vu précédemment, l'activité collective se colore aussi des productions du collectif de travail. Nous allons montrer ici combien une cohorte aïeux, grand-père, père et fils sylviculteurs peut apparaître comme un collectif de travail fécond.

\subsection{1.- Des membres liés par des phénomènes sociaux.}

$\mathrm{G}, \mathrm{P}$, L et C s'investissent dans une forêt qu'ils considèrent comme n'ayant pas été créée exclusivement pour eux, mais devant aussi bénéficier à leurs successeurs (qu'ils soient, ou non, de leur sang). Il en résulte la perception d'un rôle au sein de leur lignée, rôle qui se concrétise par des conduites de conservation, voire de développement de la forêt.

Une affection intersubjective (dans le sens d'un changement d'état d'un sujet déclenché par sa conscience de l'autre) apparaît à travers des comportements commémoratifs à formes verbales, écrites et actives. $\mathrm{P}$, par exemple, ne cesse de glorifier son père ( «le roi de la hache », «le roi des abatteurs »). Les archives de la forêt de G expriment, pour leur part, des reconnaissances posthumes. Cette affection intersubjective peut aussi avoir des répercussions plus directes sur la finalisation de l'activité, et partant, sur l'activité elle-même. P, par exemple, veut ramener du pin landais en souvenir de son père d'origine landaise, $\mathrm{C}$ 
s'abstient de couper une parcelle "parce que, effectivement, ces douglas-là c'est l'image que j'ai de mon grand-père, et j'ai le sentiment que le jour où on fera une coupe rase ça va être un crève-cœur».

Il apparaît ainsi que la lignée aïeux, grand-père, père et fils sylviculteurs n'est pas qu'une juxtaposition temporelle d'anonymes. Ce n'est pas une juxtaposition, car apparaît une structure interne reposant sur une forme de transmission que l'on peut associer à l'acte du don (Mauss, 1925) où donner, recevoir et rendre sont liés dans un système normatif. Au sein d'une configuration familiale, cet acte de transmettre s'inscrit dans une obligation « psychique » de donner et l'obligation de contractualiser une dette du côté des descendants.

D'autre part, si la lignée apparaît comme une foule d'anonymes au-delà d'un certain horizon (l'horizon des grands-parents chez L, P et C, de 7 générations de prédécesseurs chez G), les sujets semblent cependant affectés par ces «anonymes », comme l'évoque Cardon (1999). En deçà d'un horizon, la lignée apparaît comme un ensemble de membres identifiés, avec des liens sociaux. La lignée est donc davantage qu'un ensemble de sylviculteurs et présente des caractéristiques supplémentaires, comme l'exemple de $\mathrm{G}$ va le montrer.

\subsection{2- Un sentiment d'appartenance à caractère lignager}

Avec G, apparaît une «Inclusion des autres dans le Soi » (Aron, Aron, \& Smollan, 1992, cité par Richer, \& Vallerand, 1998), ce que nous prenons pour un critère de sentiment d'appartenance. Nous pouvons l'identifier dans l'usage récurrent du terme «on». Lorsque G dit «on [en parlant de certains de ses prédécesseurs] faisait des comptages » il s'annexe ses prédécesseurs, prédécesseurs qu'il a englobés en une corporation à laquelle il s'identifie. Cette situation est d'autant plus significative d'une affiliation communautaire que $G$ n'est nullement impliqué dans ces comptages puisque la règle a changé et que les comptages mentionnés ne se font plus. Ainsi, dans son cas, la cohorte aïeux, grand-père, père et fils sylviculteurs est-elle un monde social au périmètre défini et provoquant un sentiment d'appartenance. G ne s'inscrit pas dans une équipe de travail, mais dans un groupe de travail. Il devient alors intéressant de voir si ce groupe porte des «faits sociaux» à visée professionnelle, ce qui en ferait un collectif de travail.

\subsection{3.- Des faits sociaux à caractère lignager}

L'analyse des entretiens montre qu'une série d'évènements ou de faits sociaux s'imposent aux membres de la communauté lignagère et qu'ils ne sont partagés que par eux.

$\mathrm{G}$ mentionne par exemple une émotion collective lignagère : "Le vent du nord qui a balayé [en 1904] tout le fond [de la forêt] avec plus de $8000 \mathrm{~m}^{3}$ par terre et il [son aïeul d'alors] l'a écrit et je pense que ça a marqué la famille de générations en générations ». G rajoute "j'ai des doutes et je crains». Ce «souvenir collectif » n'apparaît ainsi pas neutre dans l'activité. Il s'impose à tous les membres de cette communauté lignagère (et non aux membres extérieurs).

De même G mentionne une matrice pré-organisatrice de l'action qui se transmet à tous les membres de la lignée et exclusivement à eux : «j'vois le découpage de la forêt et la gestion qu'on continue c'est vraiment lui [son aïeul vivant en 1900] qui a instauré ça; et puis c'est ancré dans, comme si c'était ancré dans les gènes de générations en générations ». On constate ainsi que le groupe lignager de G porte, pour reprendre les termes de Durkheim, ses propres «manières d'agir, de penser et de sentir [sur des registres professionnels], extérieures à l'individu, et qui sont douées d'un pouvoir de coercition en vertu duquel elles s'imposent à lui ». La lignée de $\mathrm{G}$ est un véritable collectif de travail. La question devient alors d'en évaluer sa vitalité, c'est-à-dire sa posture par rapport à la (ré)-élaboration de ses règles d'action.

\subsection{4.- Une (ré-)élaboration lignagère de règles}

La lignée de $\mathrm{G}$ présente des exemples convaincants de (ré)-élaborations collectives de règles 
à périmètre lignager. Cela peut concerner des règles de travail. Ainsi, par exemple, il existe une règle d'évaluation qui permet de diagnostiquer l'équilibre de la forêt en arbres jeunes à vieux. Cette règle, observée dans nos analyses de terrain et qui est aujourd'hui encore portée par la communauté contemporaine de métier, consiste à compter les "gros bois », "bois moyen » et «petit bois». Les archives de la forêt de G contiennent des résultats de tels comptages, ce qui prouve que cette règle avait cours dans la lignée. Mais les archives montrent le prédécesseur de $G$ remettre cette règle en cause et $G$ adhérer à cette réélaboration. Ainsi, y a-t-il cohésion lignagère autour de cette nouvelle règle, et ce en dépit de ce qui est préconisé par la communauté contemporaine de pairs. La ré-élaboration de la règle et l'adhésion collective sont bien, dans ce cas, exclusivement lignagères. Dans le cas de G donc, la communauté lignagère (aïeux, grand-père, père et fils sylviculteurs) élabore et réélabore ses propres règles de travail. Nous avons affaire à un collectif de travail «vivant ».

\subsection{5.- Formes archétypales de situations multi-générationnelles de travail sylvicole}

La lignée de $\mathrm{G}$ présente les caractéristiques de l'activité collective. Un travail collectif (réel) se combine en effet à un collectif de travail. Par ailleurs, $\mathrm{G}$ est centré sur les problématiques de préparation et de structuration de l'objet du travail (la forêt) en raison de ses effets sur l'activité. Conscient des effets de la situation de travail, il en exploite le savoir pour faciliter ou rendre plus efficientes les activités de ses successeurs. S'il prône la futaie régulière, par exemple, c'est parce que cette situation de travail facilitera son activité future, mais aussi celle de ses successeurs : "pour que quelque chose puisse être reprise et réalisée, il faut que les principes soient simples, en fait, simples et faciles à mettre en place. Une futaie irrégulière, c'est très difficile à mettre en place, une futaie régulière, c'est beaucoup plus facile».

L, en revanche, est dans une autre configuration : celle de l'activité individuelle en œuvre dans un travail collectif dans le cadre d'une communauté de métier élaborée. S'il prône la futaie régulière pour le douglas, c'est parce que cet arbre, essence de lumière, lui paraît mal s'accommoder d'une futaie irrégulière. L ne travaille pas "pour ses successeurs », pourraiton dire, il travaille d'abord "pour la forêt ». Il est centré sur l'objet du travail et son activité ne semble pas s'inscrire d'emblée dans un cadre coopératif. D'ailleurs, son groupe lignager existe peu (le «on » ou le «nous » se référant à la lignée n'apparaît qu'une fois au cours de l'entretien). Il dit ne pas avoir «pu être influencé dans [sa] gestion et [son] approche de la forêt par une tradition familiale ». À défaut de collectif lignager, $\mathrm{L}$ se repose sur les règles de métier. Dans son esprit, si tous, dans la lignée, suivent les règles élaborées par la communauté de métier, alors la forêt sera bien «entretenue». Le but est collectif, les activités des uns et des autres se coordonnent grâce à une gouvernance appropriée par règles de métiers. Un observateur extérieur peut y voir un travail lignager, mais, faute de conscience de coéquipiers de la part de L, la situation collective de travail ne s'inscrit ni dans une forme coopérative, ni même dans une forme collaborative. Le travail est « distribué ».

\section{3.- Ressources de l'organisation collective du travail}

Nous venons d'identifier 2 formes archétypales de situation multi-générationnelle de travail sylvicole : l'activité lignagère coopérative, qui se caractérise par la prise en charge d'une forêt par un collectif longitudinal, et le travail lignager distribué qui se caractérise par une mise en œuvre de tâches par des opérateurs qui ne se sentent pas impliqués dans un collectif de travail. Il nous reste maintenant à aborder la question des modes d'articulation en œuvre dans ces types d'activité.

Le premier point à examiner est celui qui concerne les modalités de l'articulation qui se traduit par l'émergence de la «visée collective» que nous avons pu observer. Mais la méthodologie que nous avons suivie ne nous permet pas d'être exhaustifs sur cette question. Il aurait en effet fallu axer nos observations sur des couples de sylviculteurs / successeurs présomptifs. 
Nous nous limitons donc à constater la réalité d'une articulation qui aboutit à une «visée collective» et nous ne nous aventurons pas sur l'étude de ses modalités d'émergence. Celles-ci semblent être à la croisée de considérations lignagères (éducation, formation) et de considérations transversales. $\mathrm{L}$ et $\mathrm{C}$ établissent en effet leurs finalités sylvicoles à partir de normes sociales contemporaines pendant que $\mathrm{P}$ et $\mathrm{G}$ semblent avoir été fortement imprégnés par leurs pères. On remarque aussi que le prédécesseur dispose d'un pouvoir : il peut forger la forêt conformément à sa visée et ainsi créer une situation qui aimante, peu ou prou, l'action de ses successeurs ${ }^{3}$.

Nous allons plus précisément aborder la question de l'articulation opérationnelle, c'est-àdire, concrètement, la question des fondements de la «bonne» coordination des actions à « visée collective » donnée. Comme nous l'avons vu, l'orchestration des actions ne s'appuie pas sur un prescrit hiérarchique, car il n'y a pas de «N+1». Cependant, deux sources de prescription semblent se dégager. Si L plante ses douglas à 1100 pieds/ha c'est parce que «la profession pense que le peuplement à onze cents plants l'hectare est la bonne densité ». La procédure est ici issue de "l'idéologie du quotidien» (Bakhtine, 1929/1977, cité par Darré, 2001). Mais lorsque nous l'interrogeons sur les consignes données pour une éclaircie, il se fait l'écho de «l'idéologie officielle», pour reprendre l'expression de Bakhtine (op. cit) qui prône l'éclaircie «dynamique».

«L'idéologie officielle» se reconnaît dans la connaissance scientifique relayée par les techniciens-conseils du CRPF et «l'idéologie du quotidien»se rapporte aux règles portées par la communauté contemporaine de métier. L déconsidère «l'idéologie officielle ». Pour lui, «les publications [i.e. l'idéologie officielle] ne font qu'entériner [l'idéologie du quotidien]». G est ouvertement plus critique à l'encontre de «l'idéologie officielle ». On constate ainsi un type de rapports que l'on trouve dans des domaines proches (domaines liés aux métiers patrimoniaux en lien avec la nature) où apparaît une cohabitation conflictuelle entre approches pragmatiques (savoirs-faire procéduraux mis en oeuvre par les paludiers, pêcheurs, éleveurs) et approches scientifiques portées par les formations scolaires, par les ingénieurs ou techniciens-conseils (Darré, 2001 ; Delbos, \& Jorion, 1984).

Ainsi, si l'orchestration des actions n'est pas le fruit d'une conception hiérarchique, elle n'est pas davantage celui d'une conception rationnelle qui s'imposerait aux acteurs.

On remarque, d'autre part, que la possibilité d'élaborer une orchestration pertinente en sylviculture n'est pas une évidence en soi, car, en raison de la durée de ses processus, la sylviculture pose une difficulté. Dans un processus court, en effet, les résultats des actions étant rapidement perçus, il est alors possible d'évaluer soi-même la pertinence des actes et ainsi de concevoir une coordination efficace des actes. Il en va différemment en sylviculture où des conséquences d'un acte peuvent apparaître quelques dizaines d'années plus tard. Cela signifie que la pertinence de l'orchestration des actions suppose une capacité multigénérationnelle - et non plus seulement individuelle - d'élaboration de l'expérience.

Finalement, la question de l'orchestration des actions en sylviculture revient d'abord à s'interroger sur les modalités d'une élaboration longitudinale de l'expérience.

\section{4.- Ressources d'organisation du travail distribué.}

Le processus sylvicole paraît rythmé par des règles de métier, du registre de la procédure, typiquement l'éclaircie, règles qui ont un statut d'incontournable dans l'esprit des sylviculteurs. À la question « pourquoi avoir fait réaliser une éclaircie? »

3 Dans les théories de l'action située, la planification est une ressource pour l'action mais n'en explique pas son cours. Le rôle des contextes matériels et culturels est premier et l'action émerge des circonstances (Béguin, \& Clot, 2004). On note qu'ici, les circonstances matérielles (la forêt) sont imprégnées des «planifications » des prédécesseurs. 
— $\mathrm{L}$ répond : «J'ai décidé de replanter, faut bien que, logiquement, je fasse ce que l'acte de planter impliquait ».

— C répond : «Ben, disons, que c'est, heu, c'est dans le, heu, c'est dans la conduite d'une plantation».

— G répond : «Alors l'éclaircie c'est, bon, alors ça c'est un principe sylvicole ».

Mais l'intériorisation du principe formel de l'éclaircie ne suffit pas. S, par exemple, observé au travail, prévoit une éclaircie. Il en a défini les grandes lignes : prélever les douglas branchus ou tordus. Mais en situation, la chose paraît plus difficile. S doit ainsi procéder à des ajustements, car, par endroits, appliquer la consigne c'est prendre le risque de «faire un trou » et de fragiliser la forêt face au vent ou de l'ouvrir au taillis. Ainsi faut-il aussi être capable de voir dans les détails ce que la forêt « demande » et agir en conséquence. Le sujet, alors positionné dans l'agir, produit un fonctionnement de type pragmatique (Pastré, 2011). Il apparaît que si certaines règles sylvicoles utilisées sont transmises oralement ou par écrit (forme prédicative de la connaissance), l'importance que semble prendre le terrain dans la formation ("On n'apprend pas sur un livre, ça s'apprend pas sur un livre»; « [J'ai appris] en allant avec mon père en forêt »; "[J'ai appris] sur l'terrain, hein, bien sur, hein, [avec mon père]»), l'importance du terrain dans la formation, donc, laisse suspecter le développement de toute une "activité cognitive sous-jacente ", "truffée d'inférences, peu accessibles à la conscience pour la plupart d'entre elles, mais qui n'en sont pas moins essentielles pour relier entre eux les éléments de l'activité» (Vergnaud, 2001, p. 5). Le sylviculteur s'appuie ainsi aussi sur la forme opératoire de la connaissance. En l'occurrence, nous avons pu constater l'écart entre la sûreté qui apparaissait dans certains des actes de $\mathrm{S}$ et ses difficultés à verbaliser précisément les raisons de ses décisions.

Ces règles non formalisées, mais guidant cependant «les façons de prendre la forêt » (connaissances opératoires) semblent communes aux gens du métier, d'où un genre professionnel. La forêt, alors, servie par un savoir pragmatique, a pris le commandement et structure les activités. C'est là un phénomène que l'on peut aussi voir dans d'autres métiers liés à la nature comme ceux de la mer, où «tout se passe comme si c'était les marais ou la mer qui dictaient comment il fallait les prendre» (Delbos, \& Jorion, 1984, p. 139). Ce phénomène est encore plus prégnant dans les cas des processus dynamiques liés au vivant, car alors «une caractéristique importante... réside dans une dialectique sujet situation, qui donne un rôle moteur important à cette dernière » (Jaunereau, 2009, p. 4).

La pertinence coordinatrice de ce savoir pragmatique peut s'expliquer par le caractère intergénérationnel de la communauté qui l'élabore.

Après avoir intériorisé les règles, explicites ou non, de leurs aînés, les générations montantes, devenues adultes remanient, après confrontation au réel, quelques savoirs prédicatifs ou opératoires. C'est le cas de L qui explique qu'autrefois «on [la communauté de pairs] avait tendance à penser qu'on pouvait planter [les douglas] avec une grosse densité comme le sapin et puis on s'est aperçu que c'était pas le cas et puis après on a pensé qu'il fallait leur donner un développement maximum et on a planté à $4 \mathrm{~m}$ ». Par ce mode d'élaboration, ces règles apparaissent comme des fruits de l'Histoire. Incognito, pourrait-on dire, la communauté élabore les leçons de son histoire et forme ainsi sa «mémoire pour prédire » (Clot, 1999), mémoire organisationnelle et collective qui, par le moyen des faits sociaux qu'elle féconde, prédispose à des activités individuelles temporellement cohérentes entre elles.

Ainsi, dans le cas du travail distribué en sylviculture, on assiste à une articulation des activités individuelles fondée sur l'adhésion à un savoir pragmatique commun, fruit d'une activité séculaire de la communauté de métier, pertinent au regard du développement d'une forêt intégrée dans son contexte. La forêt apparaît alors comme un artéfact, car porteuse «de partage et de division du travail, [avec] une signification incorporée dans une pratique sociale $\gg$ (Rabardel, 1995, p. 26). 


\section{5.- Ressources d'organisation du travail coopératif.}

L, l'archétype de l'opérateur en travail distribué, n'a pas hérité de documents et dit ne pas en avoir eu besoin, car «on a le nez dans le guidon, on regarde le terrain devant soi». Dans son cas, le travail distribué s'appuie sur la connaissance et l'application de règles indépendantes de la connaissance explicite de l'histoire autour de la forêt traitée. L'écrit n'est pas un attendu.

Ce n'est pas le cas de G pour qui la connaissance de l'Histoire est une ressource souhaitée. Il en regrette en effet un déficit : "c'est intéressant de voir ce qu'ils faisaient en 1903, parce que là, en fait, on récupère ce peuplement maintenant... [Une bonne information m'aurait été utile] dans la façon aussi de voir les parcelles où il faut intervenir ». Il ne s'agit pas là, pour lui, de se limiter à connaître l'histoire de la forêt. Il s'agit bien de connaître l'histoire autour de la forêt, c'est-à-dire l'histoire contextualisée des activités forestières en lien avec leurs effets sur la forêt.

En fait, le travail coopératif semble nécessiter une structure conceptuelle ${ }^{4}$ de trajectoire. Cela se vérifie avec S. Lors de ses discussions avec le bûcheron pour la préparation de l'éclaircie, $\mathrm{S}$ imagine une trajectoire conçue par son prédécesseur : "[Mon père] avait planté peu dense, pour éviter d'avoir à courir sans arrêt derrière des éclaircies, quitte à ce que les arbres soient un peu branchus». On identifie dans cette conception de trajectoire des éléments culturaux (rapport entre la densité de plantation et les caractéristiques futures des arbres), des finalités (le choix pour une plantation révèle une finalité «industrielle»), des buts (reboisement), des éléments contextuels (ici, le choix pour une plantation peu dense sur une grande surface prenait en compte une anticipation des ressources disponibles, extrapolait le fait qu'un arbre branchu serait plus difficilement accepté par le marché et répondait à un arbitrage entre l'état de la forêt et des attentes d'associés) et une anticipation du retentissement des actions sur le travail futur («éviter d'avoir à courir sans arrêt derrière des éclaircies »). Y est aussi sous-entendu le processus sylvicole classique mené sur un peuplement de sapins et partant, "l'organisation du travail qu'il faut déployer pour en suivre le cours, ainsi que ses effets sur les intervenants »(Strauss, 1992).

S s'inscrit dans ce schéma de trajectoire : "Ca nous a bien aidé, car on n'a pas eu besoin de s'occuper de ce coin jusqu'à maintenant. Mais maintenant il faut faire quelque chose». Mais il le développe également en explicitant son «plan»: «vu la qualité des arbres, on fera la coupe finale tôt, disons à 80 ans [c'est-à-dire dans 30 ans]. Dans 15 ans, on fait une coupe semi-définitive destinée à développer la régénération naturelle, si elle veut bien venir. Ce qui va être très important, ce sera de retirer tous les mauvais ensemenciers pour que la génération d'après soit meilleure que celle-ci ».

Ce sylviculteur se projette donc dans une trajectoire qui articule des éléments du passé (issus des choix de ses prédécesseurs), des actions du présent et des conséquences à venir pour ses successeurs. Ces aspects marquent donc bien l'esprit de coopération lignagère.

Pour autant, $\mathrm{S}$ indique aussi «s'être vu dans la coupe semi-définitive » et avoir été plus circonspect en ce qui concerne la conduite finale du reboisement par lui-même. La trajectoire entreprise ne pourra s'accomplir, après d'éventuelles révisions, que si son successeur la comprend. La coopération suppose donc une vision partagée et supposée de trajectoire. Aussi, en sylviculture, le travail coopératif lignager suppose que chaque sylviculteur puisse inscrire son action dans une vision, plus ou moins prédicative (explicitable), mais aussi opératoire (intégrant l'implicite) de trajectoire; ce qui implique une compétence du registre du savoir de trajectoire.

4 Incorporation d'un ensemble de concepts, explicites ou non, organisateurs de l'activité en une unité « cognitivement manipulable (Vidal-Gomel, \& Rogalski, 2007)». La structure conceptuelle permet au sujet de s'orienter dans une situation (Pastré, 2011). 
Mais qu'en est-il de l'élaboration collective de l'expérience nécessaire à l'élaboration d'une conception pertinente de trajectoire?

Il apparaît ici que l'écrit a un rôle capital. Pour G par exemple, «celui qui écrit le Plan Simple de Gestion, s'il reste dans cet objectif là, est obligé de comprendre les précédents, il peut pas écrire - ou bien à ce moment-là il est complètement à côté de la plaque - il peut pas écrire un Plan Simple de Gestion sans avoir fait l'effort de comprendre ce que les prédécesseurs ont écrit, en faisant cet effort-là, il intègre le fonctionnement de la forêt... il prend toute la connaissance de la forêt ». Encore faut-il que l'écrit soit «pragmatisé ». Comme le disent Grosjean et Huët (2009, p. 3), la mémoire organisationnelle par l'écrit «ne se résume pas à une tâche de recueil ou d'explicitation de savoir ou savoir-faire déjà existants, mais nécessite une (re)construction dans l'action». Comprendre un écrit est bien réussir à recréer son lien avec le réel.

L'écrit, en l'occurrence les Plans Simples de Gestion, "rend présent » son auteur «mais aussi les évènements, analyses, actions qui auront été entrepris dans un autre lieu et à un autre moment » (Grosjean, \& Huët, 2009, p. 11). D'une certaine manière, le texte, s'il est suffisamment affectant pour attirer un investissement de la part de lecteurs, a pour effet d'emporter son auteur avec lui et ainsi le transférer dans la communauté contemporaine de ses lecteurs. «Objet intermédiaire », le texte, alors, "représente ceux qui les ont conçus. Il matérialise leurs intentions, leurs habitudes de travail ou de pensée, leurs rapports et leurs interactions, leurs perspectives et les compromis qu'ils ont établis »(Vinck, 2009, p. 56), et «contribue à déplacer les points de vue des acteurs» (Op.cit, p. 59). En d'autres termes, audelà du groupe des membres en interactions, la communauté contemporaine s'élargit aux auteurs d'écrits repris et discutés dans ce groupe. Il est significatif de voir que la communauté lignagère ne dépasse pas l'horizon des grands-parents dans les lignées sans écrits ( $\mathrm{L}, \mathrm{P}$ et $\mathrm{C}$ ) alors qu'elle s'étend jusqu'à l'arrière-arrière-grand-père de $\mathrm{G}$, premier auteur d'un texte lu, relu, discuté et rediscuté. Son écrit portant sur l'aménagement de sa forêt et, partant, sur une certaine vision de trajectoire, a déclenché des processus de réflexivité (qui, parfois, se sont concrétisés par de nouveaux écrits). On pourrait dire qu'avec des écrits ayant rang d'objets intermédiaires alors le collectif lignager temporellement disjoint devient un collectif de contemporains en situation de controverses professionnelles. C'est ainsi qu'un processus séculaire, donc hors de portée de l'expérience individuelle, est ramené à la contemporanéité et nourrit ainsi une compétence individuelle en trajectoire.

Dans le cas du travail coopératif, en sylviculture, on assiste donc à une orchestration pertinente des activités individuelles fondée sur une élaboration collective (ici grâce aux écrits qui « rendent présents » leurs auteurs) d'un savoir de trajectoire.

\section{4.- Discussion et Conclusion}

La sylviculture nous place devant un cas original, rarement traité en ergonomie et en psychologie du travail. En l'occurrence celui dans lequel la durée du processus de travail dépasse la durée d'une vie humaine. Cette situation a conduit à nous intéresser à un aspect inédit: celui de l'organisation des actions en l'absence de contemporanéité des acteurs, c'est-à-dire en l'absence de « N+1 » et de possibilités d'ajustement par le geste ou la parole.

Une enquête, à partir de témoignages, de traces visibles et d'archives, accompagnée d'observations du «travail en train de se faire » a permis d'identifier deux formes de travail collectif multi-générationnel (l'activité multi-générationnelle coopérative et le travail distribué) ainsi que d'appréhender des ressources de leurs organisations collectives.

Dans une lignée, nous avons en effet vu les travaux des uns susceptibles de compléter les travaux des autres sans qu'il y ait réellement conscience d'un travail d'équipe. Ce travail distribué reposait sur l'application de règles de métier et de spécifications génériques appropriées. 
Plus précisément, dans le cas de l'activité coopérative, les sylviculteurs successifs, loin d'être des «isolés », forment un collectif de travail multi-générationnel, avec ses propres faits sociaux et règles «maison» susceptibles de ré-élaborations. Ce travail coopératif lignager s'appuie ainsi sur une vision partagée (et évolutive) de la trajectoire de travail. C'est-à-dire sur une conception pragmatique, commune et révisable qui intègre, en une seule unité «cognitivement manipulable » divers éléments, relatifs aux finalités, aux process (des manières de faire), aux conditions de réalisation, aux temporalités, aux buts, aux actions à mener (et à leurs effets) pour gérer, entretenir et transmettre le domaine forestier.

Le concept de trajectoire est un concept que l'on voit peu souvent, que l'on associe au cas du travail processuel mais dont on peut se demander s'il ne doit pas être davantage vu comme un soubassement de l'activité de conception de la tâche. Si c'est le cas, alors une vision partagée de trajectoire entre acteurs débouche sur des actions cohérentes entre elles.

Originairement, la trajectoire est une unité d'analyse qui dépasse la seule référence au process. Ce terme fusionne en effet le process avec «toute l'organisation du travail déployée à suivre ce cours, ainsi [que le] retentissement que ce travail et son organisation ne manquent pas d'avoir sur ceux qui s'y trouvent impliqués » (Strauss, 1992, p. 143). Mais, et c'est ce qui nous intéresse, la trajectoire est ici conçue dans une optique structurante car elle reste sous la tutelle d'un «pilote de trajectoire » (ici le médecin dans l'étude de Strauss). S'appuyant sur un «schéma de trajectoire », celui-ci prescrit en effet les «arcs de travail», c'est-à-dire "l'ensemble du travail qui aura besoin d'être fait » pour maîtriser le process et arriver aux objectifs (Strauss, op.cit, p. 176). Le «schéma de trajectoire » aboutit ainsi à la définition des tâches auxquelles des exécutants (ie. infirmières) devront se conformer sous l'œil de superviseurs (surveillantes).

En ce qui nous concerne, nous avons pu observer que les considérations de trajectoire sont apparues lors des phases d'exécution dans les situations de re-conception de tâches. En utilisant la terminologie de Strauss (1992), nous dirions que les sylviculteurs élaboraient des «arcs de travail » à partir de «schémas de trajectoire ». Ces derniers apparaissent donc là encore à l'origine de la tâche (ici redéfinie).

Bien entendu, toute confrontation à un process dynamique (i.e. un process qui évolue de luimême) mène naturellement au concept de «schémas de trajectoire » et ce d'autant plus que «la présence de longs délais de réponse dans les processus lents [ce qui est le cas de la forêt t renforce encore cette exigence d'anticipation » (Van Daele, \& Carpinelli, 2001, p. 3). Dans ce cas, "la compréhension de l'évolution du processus en y intégrant les effets des actions apparaît comme cruciale » (ibidem, p. 3). Mais il ne s'agit pas seulement d'intégrer les effets des actions des opérateurs. L'anticipation suppose aussi de prendre en compte les effets de tout ce qui interagit avec le process. D'un certain point de vue, c'est alors moins le process qui est vu comme dynamique que l'environnement de travail qui dépend de conditions très variables et hétérogènes (climatiques, écologiques, administratives, réglementaires, économiques).

Or, peut-on remarquer, toute situation de travail se développe aussi dans le temps. D'autre part, toute situation de travail est située dans, et par, un contexte sur lequel l'opérateur (ou un ensemble d'opérateurs) n'a qu'un contrôle partiel. Toute situation de travail confronte donc à un environnement de travail potentiellement dynamique, potentiel qui se réalise d'autant plus que la situation de travail dure dans le temps. C'est ainsi que le concept de trajectoire, et son incidence dans l'activité de conception de la tâche et, par extension, sur la coopération, pourrait s'élargir au cas du travail non processuel.

Le travail distribué en sylviculture, quant à lui, se caractérise par le fait que l'application de règles de métier et de spécifications génériques aboutisse à un travail collectif pertinent, et ce à l'insu de l'opérateur. La sylviculture nous place ainsi devant un cas d'école tout à fait intéressant. Cette situation de sylviculture confirme en effet les théories qui accordent aux collectifs des modalités auto-organisatrices «inconscientes» qui se finalisent en règles 
explicites de métier ou en règles transpersonnelles implicites (genre) qui guident, en situation de travail, les façons de «prendre les choses » (Clot, 1999).

On pourra regretter que cette étude pilote ne porte que sur un petit échantillon de propriétaires forestiers sylviculteurs qui ne permet pas la généralisation des résultats au secteur sylvicole dans son ensemble. En outre, ces personnes présentent des caractéristiques socio-biographiques et professionnelles très hétérogènes qui peuvent expliquer les manières différentes qu'elles ont d'organiser l'activité sylvivole, de s'y impliquer, voire de la transmettre aussi. Par exemple, certains (les retraités) peuvent disposer de davantage de temps pour s'engager dans les tâches de gestion de leur forêt, alors que d'autres peuvent tirer profit de leur activité professionnelle pour optimiser leurs interventions ou leurs pratiques (articulation entre les systèmes d'activité). Bien que l'analyse des conditions de réalisation de l'activité individuelle proprement dite n'ait pas été l'objectif de cette étude exploratoire (basée, rappelons-le, sur l'existence d'un collectif de travail multi-générationnel), cela mériterait néanmoins des analyses plus fouillées pour cerner les possibles incidences de ces facteurs socio-professionnels dans la transmission.

D'autre part, les propriétaires forestiers sylviculteurs interrogés ou observés présentent des caractéristiques qui les éloignent des cas habituellement traités en ergonomie ou en psychologie du travail (propriété de biens hérités, activité auxiliaire, enjeux financiers particuliers...). Dès lors, on peut s'interroger sur la portée de ces résultats sur d'autres secteurs d'activité. Enfin, les questions de transmission générationnelle contemporaine (entre membres d'une même famille ainsi qu'avec les acteurs de la gestion forestière) devraient être davantage explorées, notamment pour déterminer le rôle que ces personnes peuvent jouer dans l'organisation de l'activité et de sa transmission.

Ces résultats restent donc à confirmer et à enrichir par une étude de plus grande ampleur.

\section{5.- Glossaire}

CRPF : Centre Régional de la Propriété Forestière. Il s'agit d'un organisme public chargé de développer et d'orienter la production des forêts privées.

Débardage : opération qui consiste à transporter les grumes de leur lieu d'abattage à une zone de stockage en bord de route.

Dépressage : $1^{\text {ère }}$ éclaircie sur un jeune peuplement.

Éclaircie : Opération sylvicole consistant à couper, régulièrement, des arbres avant leur terme, typiquement pour favoriser le développement des arbres restants.

Futaie irrégulière : Type d'aménagement qui, au sein d'une parcelle, étage des générations différentes de bouquets d'arbres de même âge.

Futaie régulière : Les arbres occupant une même parcelle ont approximativement le même âge.

Marquage : désignation par une marque (par exemple de peinture) d'arbres à prélever (ou à conserver).

Taillis : bois d'arbres de petites dimensions.

\section{6.- Bibliographie}

Anzieu, D., \& Martin, J.Y. (1968). La dynamique des groupes restreints (6 ${ }^{\text {ième }}$ édition). Paris: PUF.

Barthe, B., \& Quéinnec, Y. (1999). Terminologie et perspectives d'analyse du travail collectif en ergonomie [version électronique]. L'Année Psychologique, 4, 663-686.

Béguin, P., \& Clot, Y. (2004). L'action située dans le développement de l'activité [version électronique]. Activités, 1(2), 27-49.

Brun, M., \& Mallein, Ph. (2009). Une recherche sur les profils d'identité située des propriétaires forestiers privés dans la relation à leurs bois, à leur forêt : profils de valeurs et acceptabilité de 
propositions innovantes pour la mobilisation de la ressource bois ; focus sur le bois-énergie. Importation le 6 mars 2011 de www.tpr.asso.fr/Documents/Documents/profilspropri\%C3\%A9taires\%20forestiers.pdf.

Cardon, Ph. (1999). Un capital dormant. La transmission patrimoniale de la forêt paysanne en FranceComté. Terrain. Importation le 8 mars 2010 de http://terrain.revues.org/index 2775.html

Caroly, S. (2010). L'activité collective et la réélaboration des règles : des enjeux pour la santé au travail. Importation le 22 octobre 2010 de http://tel.archivesouvertes.fr/docs/00/46/50/89/PDF/HDR_Caroly.pdf

Cellier, J.M., \& Marquié, J.C. (1980). Système d'activités et régulations dans l'exploitation agricole. Le Travail humain, 43(2), 321-336.

Cerf, M., \& Meynard, J.M. (2006). Les outils de pilotage des cultures : diversité de leurs usages et enseignements pour leur conception. Natures Sciences Sociétés, (2006/1), 19-29.

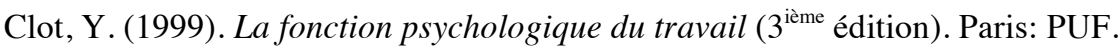

Darré, J.P. (2001). Veaux bretons et brebis alpines, entre objectivisme abstrait et relativisme [version électronique]. Travailler, (2/6), 89-103.

Delbos, G., \& Jorion, P. (1984). La transmission des savoirs. Paris: Éditions de la Maison des sciences de l'homme.

Durkheim, E. (1895). Les règles de la méthode sociologique. Paris: Flammarion.

Grosjean, S., \& Huët, R. (2009). Ecritures, textes et mobilisation des connaissances : le cas des carnets de terrain [version électronique]. Actes du Congrès annuel de l'Association des sciences administratives du Canada, $(30,22)$.

Grosjean, M., \& Lacoste, M. (1999). Communication et intelligence collective : Le travail à l'hôpital. Paris: PUF.

Hoc, J. M., \& Leplat, J. (1983) Evaluation of different modalities of verbalization in a sorting task. International Journal of Man-Machine Studies, 18, 283-306.

Jaunereau, A. (2009). Professionnalisation et apprentissage à l'aide d'un simulateur dans un environnement dynamique lié au vivant : apprendre à mettre en place une culture de colza. Travail et apprentissage, $4,13-25$.

Leplat, J. (2011). Mélanges ergonomiques : activité, compétence, erreur. Toulouse: Octarès Éditions.

Mauss, M. (1925). Essai sur le don. Forme et raison de l'échange dans les sociétés archaïques [version électronique]. L'Année Sociologique, seconde série, 1923-1924, tome I.

Molinier, P. (2006). Les enjeux psychiques du travail. Paris: Payot \& Rivages.

Pastré, P. (2011). La didactique professionnelle : Approche anthropologique du développement chez les adultes. Paris: PUF.

Pueyo, V., \& Zara-Meylan, V. (2012). Impacts d'outils de gestion sur la conduite de cultures en pépinière. Activités, (9/1), 1-20

Rabardel, P. (1995). Les hommes et les technologies. Paris: A. Colin.

Richer, S., \& Vallerand, R. (1998). Construction et validation de l'Echelle du sentiment d'appartenance sociale [version électronique]. Revue Européenne de Psychologie Appliquée, 48(2), 129-137.

Schepens, F. (2007). Hommes des bois? Socio-anthropologie d'un groupe professionnel. Paris: CTHS.

Schmidt, K. (2002). The problem with awareness. Computer Supported Cooperative Work: The Journal of Collaborative Computing, 11(3-4), 285-298.

Strauss, A. (1992). La trame de la négociation : Sociologie qualitative et interactionnisme. Paris: L'Harmattan.

Valléry, G., \& Amalberti, R. (2006). L'analyse du travail en perspectives, Influences et évolution. Toulouse: Octarès Éditions.

Van Daele, A., \& Carpinelli, F. (2001). La planification dans la gestion des environnements dynamiques : quelques apports récents de la psychologie ergonomique. Importation le 15 juillet 2011 de http://w3.umh.ac.be/pub/ftp_psytrav/PDF/Plani_env_dyna.pdf.

Vergnaud, G. (2001). Forme opératoire et forme prédicative de la connaissance. Actes du colloque 
GDM 2001. Importé le 18 mars 2011 de http://smf4.emath.fr/Enseignement/ TribuneLibre/EnseignementPrimaire/ConfMontrealmai2001.pdf

Vidal-Gomel, Ch., \& Rogalski, J. (2007). La conceptualisation et la place des concepts pragmatiques dans l'activité professionnelle et le développement des compétences. [version électronique]. Activités, 4(1), 49-84.

Vinck, D. (2009). De l'objet intermédiaire à l'objet frontière. Vers la prise en compte du travail d'équipement. [Version électronique]. Revue d'anthropologie des connaissances, 3(1), 51-72.

\section{RESUME}

Nous intéressant au cas de la sylviculture nous y montrons la réalité d'une situation collective de travail multi-générationnel de conduite de forêts. Cette situation collective de travail apparaît sous 2 formes: la forme du travail distribué et celle de l'activité coopérative longitudinale. Dans la forme du travail distribué, les sylviculteurs appliquent des règles de métier, explicites ou non, collectivement élaborées dans, et par, l'Histoire. Dans la forme de l'activité coopérative, les sylviculteurs exploitent, en connaissance de causes, des savoirs sur le fonctionnement de leur forêt pour optimiser la situation de travail future et ainsi faciliter les activités de leurs successeurs. Dans ce cas, la coopération s'appuie sur une conception pragmatique et partagée de trajectoire, conception qui se construit dans une forme de controverse entretenue par la médiation de documents non contemporains les uns des autres et devenus objets intermédiaires.

\section{MOTS-CLES}

sylviculture, collectif de travail, travail collectif, trajectoire

\section{REFERENCEMENT}

Bobillier Chaumon, M.-E., Ruyneau de Saint George, P., Cuvillier, B., \& Sarnin, Ph. (2014). Sylviculture et activité collective longitudinale : un premier repérage. Activités, 11(1), 3-21, http://www.activites.org/v11n1/v11n1.pdf

Article soumis le 8 janvier 2012, accepté pour publication le 26 septembre 2013. 